\title{
Dienogest exerts its anti-endometriotic effect throughthe direct suppression of matrix metallopeptidases
}

Hiroshi Honda ( $\nabla$ h-honda@qg8.so-net.ne.jp )

Hiroshima City Asa Hospital https://orcid.org/0000-0003-0943-6583

Norihisa Nishimichi

Hiroshima University

Michinori Yamashita

Fujian Medical University

Yumiko Akimoto

Sumire Women's Clinic

Hirotoshi Tanimoto

Sumire Women's Clinic

Mitsue Teramoto

Sera Central Hospital

Hideki Teramoto

Sera Central Hospital

Yasuyuki Yokosaki

Hiroshima University

\section{Research}

Keywords: endometriosis, dienogest, microarray, gene expression profile, ingenuity pathways analysis, MMP

Posted Date: October 2nd, 2020

DOI: https://doi.org/10.21203/rs.3.rs-84809/v1

License: (c) (1) This work is licensed under a Creative Commons Attribution 4.0 International License.

Read Full License 
3 Dienogest exerts its anti-endometriotic effect through

\section{the direct suppression of matrix metallopeptidases}

6 Hiroshi Honda ${ }^{1 *}$, Norihisa Nishimichi ${ }^{2}$, Michinori Yamashita ${ }^{1,3}$, Yumiko Akimoto $^{1,4}$, Hirotoshi Tanimoto ${ }^{1,4}$, Mitsue Teramoto ${ }^{1,5}$, Hideki Teramoto ${ }^{1,5}$, and Yasuyuki Yokosaki ${ }^{2}$

1: Department of Obstetrics and Gynecology, Hiroshima City Asa Hospital

2: Cell-Matrix Frontier Laboratory, Health Administration Center, Hiroshima University

3 Department of Surgery and Palliative Medicine, Fujita Health University School of Medicine

4: Sumire Women's Clinic

5: Department of Obstetrics and Gynecology, Sera Central Hospital

*Corresponding author: Hiroshi Honda, Department of Obstetrics and Gynecology, 


\section{Abstract}

2 Background: Endometriosis, which affects up to $10 \%$ women of reproductive age, is

3 defined by the presence of ectopic endometrial tissue outside the uterus. The current key

4 drug of hormonal therapies for endometriosis is dienogest, which is a progestin with

5 high specificity for the progesterone receptor. Although many findings about the anti-

6 endometriotic effect of dienogest on endometriosis have been reported, the precise

7 mechanisms of dienogest's anti-endometriotic effect remain unknown.

8 Methods: To investigate the direct anti-endometriotic effect of dienogest on

9 endometriotic cells, we determined and compared the genome-wide gene expression

10 profiles of endometriotic stromal cells treated with dienogest (Dienogest group) and

11 those not treated with dienogest (Control group) and then performed a pathway analysis

12 using these data. To test the microarray data, we performed real-time RT-PCRs for

13 matrix metallopeptidase (MMP)-1, MMP-3, MMP-10, and TIMP-4.

14 Results: Six-hundred forty-seven genes were revealed to be differentially expressed

15 between the Dienogest and Control groups. Of them, 314 genes were upregulated and

16333 genes were downregulated in the Dienogest group compared to the Control group.

17 We identified 20 canonical pathways that are significantly distinct in the Dienogest

18 group versus the Control group. Among the 20 canonical pathways, MMPs including

19 MMP-1, -3, and -10 were found to be the most involved genes.

20 Conclusions: Our results suggest that dienogest may exert its anti-endometriotic effect

21 through the direct suppression of MMPs.

23 Key words: endometriosis, dienogest, microarray, gene expression profile, ingenuity

24 pathways analysis, MMP 


\section{Background}

Endometriosis, which is defined by the presence of ectopic endometrial tissue outside the uterus, is a common chronic disease affecting up to $10 \%$ women of reproductive age [1]. Infertility and chronic pelvic pain are the main symptoms. For chronic pelvic pain, mainly hormonal therapies have been provided in the past decades, and a gonadotropin-releasing hormone $(\mathrm{GnRH})$ analogue has been used as the key drug of the hormonal therapies. However, a GnRH analogue cannot be used for a long period because of its side effects such as bone loss. It has thus been difficult for individuals with chronic pelvic pain due to endometriosis to obtain relief from pain over a long period by using a GnRH analogue.

Dienogest is classified as a 4th-generation progestin with high specificity for the progesterone receptor. It has an effect on endometriosis-induced pain that is equivalent to that of a GnRH analogue. However, unlike GnRH analogues, dienogest can be used for long periods, and it has brought endometriosis patients long-term relief from the pain [2]. Dienogest has replaced GnRH analogues as the key drug of the hormonal therapies for endometriosis. The anti-endometriotic effect of dienogest is thought to be due mainly to its ability to suppress ovulation [3], and several studies have shown that dienogest directly inhibits the inflammatory responses or aromatase expression in endometriotic cells $[4,5,6]$. Another investigation indicated that dienogest directly inhibits the proliferation of endometriotic stromal cells [7]. Although many studies have attempted to reveal the biological mechanisms that underly the effects of dienogest on endometriosis, the precise mechanisms remain unknown.

Genome-wide gene expression profiling using a microarray and its subsequent pathway analysis sometimes reveal novel biological cell-related findings for a disease. 
1 There has been no report of genome-wide gene expression profiling conducted to

2 investigate the biological actions of dienogest in endometriotic cells.

\section{Methods}

The present study analyzed the genome-wide gene expression profile of endometriotic stromal cells treated with dienogest. We also performed a pathway analysis using the data of the gene expression profile. The results demonstrated that in the endometriotic stromal cells, matrix metallopeptidases (MMPs) were the most closely involved genes among the genes that are directly modified by dienogest.

\section{Patients and samples}

The protocol of the present study was approved by the Ethical Committee of Hiroshima City Asa Hospital, and all experiments were carried out in accordance with relevant guidelines and regulations. Tissue specimens of ovarian endometriotic cysts $(n=5)$ were obtained during gynecological surgeries from five patients with stage III-IV endometriosis evaluated according to the American Society for Reproductive Medicine classification of endometriosis. Before the surgeries, the patients provided their written informed consent. All five patients were of reproductive age and had not received any hormonal therapies. All specimens were collected at the proliferative phase of the menstrual cycle and were pathologically confirmed as endometriotic cyst tissues after the surgeries.

The isolation of endometriotic stromal cells (ESCs) was performed as described by Honda et al [8]. Briefly, Endometriotic tissue layers were scraped from the inner wall of the cyst, minced into small pieces, and enzymatically dissociated by incubation with 
$1 \quad 0.25 \%$ collagenase (Sigma-Aldrich, St. Louis, MO, USA) and 0.02\% DNase I (Sigma-

2 Aldrich) in phenol-red-free Dulbecco's modified Eagle's Medium/Ham's F-12

3 (Invitrogen, Carlsbad, CA) supplemented with 10\% charcoal-stripped fetal bovine

4 serum (FBS) (Invitrogen) for $1 \mathrm{hr}$ at $37^{\circ} \mathrm{C}$ in an atmosphere of $5 \% \mathrm{CO}_{2}$. Enrichment of

5 the ESCs was performed by serial filtration using $100-\mu \mathrm{m}$ and $40-\mu \mathrm{m}$ nylon sieves (BD

6 Falcon, Franklin Lakes, NJ), and equally collected onto two 6-cm culture dishes per

7 sample. After incubation at $37^{\circ} \mathrm{C}$ for $30 \mathrm{~min}$ to allow the ESCs to attach to the dishes,

8 the media were removed to remove the floating endometriotic epithelial cells in the

9 supernatant.

10 The ESCs were cultured in DMEM/Ham's F-12 medium supplemented with 10\%

11 charcoal-stripped FBS and $1 \%$ penicillin and streptomycin $(100 \mathrm{mg} / \mathrm{ml})$ (Invitrogen)

12 under the conditions described above. The medium was changed every other day, and,

13 when the cells reached $80 \%$ confluence, the cultures were serum-starved in serum-free

14 DMEM/Ham's F-12 medium before hormone treatments. After $24 \mathrm{hr}$ of culture, the

15 medium was replaced with either serum-free DMEM/Ham's F-12 medium with estradiol

$16\left(10^{-8} \mathrm{M}\right.$; Sigma-Aldrich) alone or estradiol $\left(10^{-8} \mathrm{M}\right)+\operatorname{dienogest}\left(10^{-6} \mathrm{M}\right.$; Santa Cruz

17 Biotechnology, Dallas, TX). The ESCs treated without dienogest were used as a control

18 (Control group), and those treated with dienogest were named the Dienogest group.

19 After these hormonal treatments for $48 \mathrm{hr}$, the ESCs were directly lysed on the culture

20 dishes with TRIzol ${ }^{\mathrm{TM}}$ reagent (Invitrogen), immediately snap-frozen, and stored at

$21-80^{\circ} \mathrm{C}$ until further processing.

RNA isolation

Total RNA was purified from the cell lysates of ESCs using the RNeasy Mini Kit 
1 (Qiagen, Valencia, CA) according to the manufacturer's instructions. The quantity and

2 quality of the purified RNA were measured and assessed using a Nanodrop ND-1000

3 spectrophotometer (Thermo Fisher Scientific, Waltham, MA) and an Agilent

4 Bioanalyzer (Agilent Technologies, Santa Clara, CA) before cRNA amplification and

5 labeling.

6

7 The following processes of cRNA amplification and labeling, sample hybridization,

8 and data analysis of the microarray were performed according to the protocol described

9 by Yokoi et al [9]. with minor modification.

cRNA amplification and labeling

Total RNA was amplified and labeled with cyanine 3 (Cy3) using the Agilent

Low Input Quick Amp Labeling Kit, one-color (Agilent Technologies) following the manufacturer's instructions. Briefly, $100 \mathrm{ng}$ of total RNA was reversed transcribed to double-strand cDNA with the use of a poly dT-T7 promoter primer. The primer, template RNA, and quality-control transcripts of known concentration and quality were

17 first denatured at $65^{\circ} \mathrm{C}$ for $10 \mathrm{~min}$ and incubated for $2 \mathrm{hr}$ at $40^{\circ} \mathrm{C}$ with $5 \mathrm{X}$ first strand

18 Buffer, 0.1 M DTT, 10 mM dNTP mix, and AffinityScript RNase Block Mix. The

19 AffinityScript enzyme was inactivated at $70^{\circ} \mathrm{C}$ for $15 \mathrm{~min}$.

The cDNA products were then used as templates for in vitro transcription to generate fluorescent $\mathrm{cRNA}$. The cDNA products were mixed with a transcription master mix in the presence of T7 RNA polymerase and Cy3 labeled-CTP and incubated at $40^{\circ} \mathrm{C}$ for $2 \mathrm{hr}$. Labeled cRNAs were purified using Qiagen's RNeasy mini-spin columns and eluted in $30 \mu 1$ of nuclease-free water. After amplification and labeling, the cRNA 
1 quantity and the incorporation of cyanine were determined using the Nanodrop ND-

21000 spectrophotometer and the Agilent Bioanalyzer.

3

\section{Sample hybridization}

For each hybridization, $0.60 \mu \mathrm{g}$ of Cy3-labeled cRNA was fragmented and then hybridized at $65^{\circ} \mathrm{C}$ for $17 \mathrm{hr}$ with an Agilent SurePrint G3 Human GE v2 8x60K Microarray (Design ID: 039494). After washing, the microarrays were scanned using an Agilent DNA microarray scanner.

\section{Data analysis of the microarray}

The intensity values of each scanned feature were quantified using Agilent feature extraction software ver. 10.7.3.1, which performs background subtractions. We used only features that were flagged as 'no errors' (present flags), and we excluded features that were not positive, not significant, not uniform, not above the background, saturated, or population outliers (marginal and absent flags). Normalization was performed using Agilent GeneSpring GX ver. 11.0.2 software (per chip: normalization to the 75th percentile shift; per gene: normalization to the median of all samples). There are a total of 50,599 probes on the Agilent SurePrint G3 Human GE v2 8x60K Microarray (Design ID: 039494) without control probes. The RNA samples of the Control group were used as the total RNA reference.

Differentially expressed genes between the Dienogest and Control groups with corrected p-values $<0.05$ and an absolute fold change $>1.5$ were considered to be significantly differentially expressed. The microarray data were also used for an ingenuity pathway analysis (IPA, Ingenuity ${ }^{\circledR}$ Systems, www.ingenuity.com) for the analysis of 
1 canonical pathways. Fisher's exact test was used to calculate a p-value to determine the

\section{Results} al [11].

\section{Results} groups significance of each canonical pathway, and p-values $<0.05$ were accepted as significant.

\section{Quantitative RT-PCR}

Total RNA purified for microarray was also used for quantitative real-time reverse transcription-polymerase chain reaction (RT-PCR) to validate the microarray data. One microgram of total RNA was reverse transcribed into first-strand cDNA by using a first-strand cDNA synthesis kit, ReverTra Ace- $\alpha$ (Toyobo, Osaka, Japan) with random primers. The cDNA was stored at $-20^{\circ} \mathrm{C}$ until used.

\section{Of the genes that were differentially expressed between the Dienogest and} Control groups, those for MMP-1, MMP-3, MMP-10, and TIMP-4 were selected for the PCR reactions, which were performed in an ABI 7300 Real Time PCR System (Applied Biosystems, Carlsbad, CA) using the KAPA SYBR ${ }^{\circledR}$ FAST qPCR kit (Nippon Genetics, Tokyo) under thermal cycling conditions according to the manufacturer's instructions. The primer sequences of the MMPs and TIMP-4 used in the analysis were the same as those designed by Pennington [10]. A real-time RT-PCR for the housekeeping gene GAPDH was performed in all of the samples to evaluate the quality of the cDNAs used in the study. The primers used for the GAPDH quantification were designed by Wang et

Identification of genes differentially expressed between the Dienogest and Control

Under the condition of a fold change of at least \pm 1.5 with corrected $p$-values of 
$<0.05,647$ genes were revealed to be differentially expressed between the Dienogest and Control groups. Of these 647 genes, 314 genes were upregulated and 333 genes were downregulated in the Dienogest group compared to the Control group. Tables 1 and 2 show the top 20 genes upregulated or downregulated in the Dienogest group, respectively.

\section{Ingenuity pathways analysis (IPA)}

The comparison analysis performed by IPA for the Dienogest and Control groups identified 20 significant canonical pathways based on these 647 differentially expressed genes, which were extracted under the same conditions. Figure 1 shows the top 10 pathways of those 20 canonical pathways. The most frequently associated genes in the 20 canonical pathways were MMPs (MMP-1, -3, -10, -12, -25, and -27). Table 3 shows the eight canonical pathways with which these MMPs are associated among the 20 canonical pathways.

\section{Quantitative RT-PCR for the validation of the microarray data}

Of the genes that were revealed to be differentially expressed between the Dienogest and Control groups, we selected MMP-1, -3, and -10 and TIMP-4 for validation of the microarray data. The data illustrated in Figure 2 demonstrated that MMP-1, -3 , and -10 were significantly decreased in the the Dienogest group compared to the Control group, and that TIMP-4 was significantly increased in the Dienogest group compared to the Control group. 


\section{Discussion}

Although dienogest has already been used for the main hormonal therapy of endometriosis, its precise anti-endometriotic mechanism has been unclear. In the present study, we comprehensively investigated the direct effects of dienogest on the endometriotic stromal cells, and our findings revealed that the MMPs were the most frequently involved genes in the 20 canonical pathways that are significantly different between the Dienogest and Control groups. Our results thus suggest that MMPs have central roles in the anti-endometriotic effect of dienogest.

Surprisingly, although several investigations have described an antiendometriotic effect of dienogest, there has been no report such as the present study that analyzed differences in genome-wide gene expression profiles between untreated endometriotic cells and endometriotic cells treated with dienogest. Bruner et al [12]. reported that the establishment of ectopic human endometrium in nude mice was inhibited by the suppression of MMPs caused by progesterone treatment. In addition, abnormal expressions of specific MMPs have been shown in endometriotic lesions compared to eutopic endometrium. Several research groups have observed that endometriotic cells have significantly higher expressions of MMP-1, -2, -3, -7, or -9 compared to eutopic endometrial cells in in vitro or in vivo experiments, or in patients' tissues[13, 14, 15, 16, 17, 18, 19]. We thus believe that MMPs play crucial roles in the establishment and progress of endometriotic lesions.

In the present study, the expressions of MMP-1 and MMP-3 in ESCs were downregulated by the addition of dienogest. Earlier studies noted that MMP-1 and MMP-3 were inhibited by progesterone or progestin in endometrial explants or endometriotic cells [20, 21, 22]. The downregulations of MMP-1 and MMP-3 by 
1 dienogest are thus likely to be reliable. The expression of MMP-7, which in

2 endometrium or endometriotic tissues is expressed only in epithelial cells, has been

3 shown to be inhibited by progesterone or progestin in two studies [21 22], but our

4 present investigation did not reveal the similar effect of dienogest on the endometriotic

5 cells. This discrepancy between the present and past studies regarding the effect of

6 dienogest is probably due to the use of endometriotic stromal cells in the present study.

$7 \quad$ It therefore seems that dienogest possibly has an inhibitory effect on MMP-7 expression

8 in endometriotic epithelial cells.

9 Our present analyses did not reveal any inhibitory effect of dienogest on the expression of MMP-2 or MMP-9. Regarding the inhibitory effect of progesterone on MMP-2 or MMP-9 expression, conflicting results have been reported. Some studies indicated that progesterone inhibited MMP-2 or MMP-9 expression in endometriotic cells, whereas other studies indicated that progesterone did not change the MMP-2 or MMP-9 expression [23, 24]. It was also reported that the MMP-10 expression in the endometriotic tissues of ovarian endometriosis was significantly increased compared to the expression in eutopic endometrial tissues without endometriosis [25], and our present results demonstrated that dienogest directly inhibited the MMP-10 expression in the endometriotic stromal cells. Taken together, the previous and present findings indicate that the suppression of MMP-10 expression (as well as those of MMP-1 and MMP-3) by dienogest might be one of dienogest's direct anti-endometriotic effects. We also observed changes in the expressions of MMP-12, -25 , and -27 by the addition of dienogest, but the involvement of these MMPs in the pathogenesis of endometriosis has not been well studied. It remains unknown whether the changes in the expressions of these MMPs are related to the anti-endometriotic effect of dienogest. 
Tissue inhibitors of metalloproteinases (TIMPs) have also been revealed to be abnormally expressed in endometriotic tissues compared to normal eutopic endometrium. The expressions of TIMP-1, -2 , and -3 were reported to be significantly lower in endometriotic tissues than normal eutopic endometrium [26, 27], and an experimental study showed that the addition of TIMP protein to the peritoneal cavity of nude mice prevented the establishment of endometriosis [12]. These findings indicate that TIMPs are also likely to be involved in the pathogenesis of endometriosis.

However, there are few studies describing a change in the expression of TIMP-1, -2 , or -3 by an addition of progesterone, and our present findings did not provide any information about the changes of TIMP expressions by the addition of dienogest, with the exception of TIMP-4. TIMP-4, which is also expressed in human endometrium, has affinity for MMP-1, $-2,-3,-7,-9$, and -12 and shows the highest inhibitory capacity for MMP-26 [28, 29, 30, 31]. TIMP-4 is coordinately expressed with MMP-26, which is an effective activator of pro-MMP-9 [32]. The results of our present analyses showed that dienogest increased the expression of TIMP-4 in endometriotic stromal cells. Although there has been no report about the relationship between endometriosis and TIMP-4, the increase in TIMP-4 expression by treatment with dienogest may be related to the antiendometriotic effect of dienogest.

This study has some limitations to consider. Endometriotic tissues consist of various types of cells such as ectopic endometrial epithelial and stromal cells and immune cells. Inflammatory cytokines, which are produced mainly by immune cells in endometriotic tissues, are thought to play important roles in the pathogenesis of endometriosis [33]. However, the experimental system of the present study lacked immune cells, and it was thus not possible to assess the anti-endometriotic effect of 
1 dienogest through the inflammatory cytokines in this study, which used only

2 endometriotic stromal cells.

\section{Conclusions \\ Conclusions} conceive.

It is also not yet possible to recreate the exact biological environment of endometriotic tissues in an in vitro experiment such as that in the present study; however, our findings revealed for the first time the direct effect of dienogest on endometriotic stromal cells by genome-wide gene expression profiling and a pathway analysis. MMPs were most frequently involved in the canonical pathways distinct to the dienogest-treated endometriotic stromal cells. Our results strongly suggest that MMPs play important roles in the therapeutic action of dienogest against endometriosis.

Although our findings demonstrated the direct suppression of MMPs by dienogest in endometriotic stromal cells, we were not able to discern the precise molecular pathways that link dienogest to MMPs. It is certain that dienogest is useful and effective for endometriosis, but it is not used for patients who want to conceive, because of its antiovulatory effect. Patients with severe endometriosis who want to conceive more than once are likely to experience disease progression during under the period without dienogest. A substrate that suppresses the expressions of MMPs at a region downstream from dienogest and that does not suppress ovulation is desired, as such a substrate could conceivably be used as a drug to treat patients with endometriosis who want to

It is clear from many earlier investigation that MMPs play important roles in the pathogenesis of endometriosis and that their expressions are regulated mainly by inflammatory cytokines $[12-19,33]$. We speculated that dienogest might indirectly 
1 suppress the expressions of MMPs through the inhibition of inflammatory cytokines

2 [33]. Our present findings suggest that in addition to its indirect suppression, dienogest

3 may exert its anti-endometriotic effect through the direct suppression of MMPs.

4

6 Availability of data and materials

$7 \quad$ The datasets used and analyzed during the current study are available from the

8 corresponding author on reasonable request.

9

10 Abbreviations

11 MMP: matrix metallopeptidases

12 GnRH: gonadotropin-releasing hormone

$13 \quad$ ESCs: endometriotic stromal cells

$14 \quad$ IPA: Ingenuity pathways analysis

15 TIMPs: Tissue inhibitors of metalloproteinases

\section{References}

1. Shafrir AL, Farland LV, Shah DK, Harris HR, Kvaskoff M, Zondervan K, et al. Risk for and consequences of endometriosis: A critical epidemiologic review. Best Pract Res Clin Obstet Gynaecol 2018;51:1-15.

2. Römer T. Long-term treatment of endometriosis with dienogest: retrospective analysis of efficacy and safety in clinical practice. Arch Gynecol Obstet. 2018;298(4):747-53. 
1 3. Moore C, Carol W, Gräser T, Mellinger U, Walter F. Influence of dienogest on

2 ovulation in young fertile women. Clinical Drug Investigation 1999; 18:271-8.

3 4. Klipping C, Duijkers I, Faustmann TA, Klein SF, Schuett B. Pharmacodynamic

$4 \quad$ study of four oral dosages of dienogest. Fertil Steril. 2010;94:S181.

5 5. Grandi G, Mueller M, Bersinger NA, Cagnacci A, Volpe A, McKinnon B. Does

6 dienogest influence the inflammatory response of endometriotic cells? A systemic

7 review. Inflamm Res. 2016;65:183-92

8 6. Shimizu Y, Mita S, Takeuchi T, Notsu T, Mizuguchi K, Kyo S. Dienogest, a

9 synthetic progestin, inhibits prostaglandin E2 production and aromatase expression

10 by human endometrial epithelial cells in a spheroid culture system. Steroids.

$112011 ; 76: 60-7$.

7. Fu L, Osuga Y, Morimoto C, Hirata T, Hirota Y, Yano T, et al. Dienogest inhibits BrdU uptake with G0/G1 arrest in cultured endometriotic stromal cells. Fertil Steril. 2008;89:1344-7.

8. Honda, H, Barrueto, FF, Gogusev, J, Im, DD, Morin, PJ. Serial analysis of gene expression reveals differential expression between endometriosis and normal endometrium. Possible roles for AXL and SHC1 in the pathogenesis of

9. Yokoi A, Yoshioka Y, Yamamoto Y, Ishikawa M, Ikeda SI, Kato T, et al. Malignant extracellular vesicles carrying MMP1 mRNA facilitate peritoneal dissemination in ovarian cancer. Nat Commun. 2017;8:14470.

10. Pennington, C. Real-time PCR expression profiling of MMPs and TIMPs. Methods in molecular biology. 2010;622:159-73. 
1 Matrix metalloproteinase 2 (MMP2) and MMP9 secreted by erythropoietin-activated

2 endothelial cells promote neural progenitor cell migration. J Neurosci. 2006;26:599636003.

12. Bruner KL, Matrisian LM, Rodgers WH, Gorstein F, Osteen KG. Suppression of matrix metalloproteinases inhibits establishment of ectopic lesions by human endometrium in nude mice. J Clin Invest. 1997;99:2851-7.

13. Kokorine I, Nisolle M, Donnez J, Eeckhout Y, Courtoy PJ, Marbaix E. Expression of interstitial collagenase (matrix metalloproteinase-1) is related to the activity of human endometriotic lesions. Fertil Steril. 1997;68:246-51.

14. Hudelist G, Keckstein J, Czerwenka K, Lass H, Walter I, Auer M, et al. Estrogen receptor beta and matrix metalloproteinase 1 are coexpressed in uterine endometrium and endometriotic lesions of patients with endometriosis. Fertil Steril. 2005;84:1249-56.

15. Ueda M, Yamashita Y, Takehara M, Terai Y, Kumagai K, Ueki K, et al. Gene expression of adhesion molecules and matrix metalloproteinases in endometriosis. Gynecol Endocrinol. 2002;16:391-402.

16. Cox KE, Piva M, Sharpe-Timms KL. Differential regulation of matrix metalloproteinase-3 gene expression in endometriotic lesions compared with endometrium. Biol Reprod. 2001;65:1297-1303.

17. Gilabert-Estellés J, Estellés A, Gilabert J, Castelló R, España F, Falcó C, et al. Expression of several components of the plasminogen activator and matrix metalloproteinase systems in endometriosis. Hum Reprod. 2003;18:1516-22.

18. Chung HW, Wen Y, Chun SH, Nezhat C, Woo BH, Lake Polan M. Matrix metalloproteinase- 9 and tissue inhibitor of metalloproteinase- 3 mRNA expression in 
1 ectopic and eutopic endometrium in women with endometriosis: A rationale for

2 endometriotic invasiveness. Fertil Steril. 2001;75:152-9.

3 19. Pitsos M, Kanakas N. The role of matrix metalloproteinases in the pathogenesis of endometriosis. Reprod Sci. 2009;16:717-22

20. Vassilev V, Pretto CM, Cornet PB, Delvaux D, Eeckhout Y, Courtoy PJ, et al. Response of matrix metalloproteinases and tissue inhibitors of metalloproteinases messenger ribonucleic acids to ovarian steroids in human endometrial explants mimics their gene- and phase-specific differential control in vivo. J Clin Endocrinol Metab. 2005;90:5848-57.

21. Bruner-Tran KL, Eisenberg E, Yeaman GR, Anderson TA, McBean J, Osteen KG. Steroid and cytokine regulation of matrix metalloproteinase expression in endometriosis and the establishment of experimental endometriosis in nude mice. $\mathrm{J}$ Clin Endocrinol Metab. 2002;87:4782-91.

22. Bruner-Tran KL, Zhang Z, Eisenberg E, Winneker RC, Osteen KG. Downregulation of endometrial matrix metalloproteinase-3 and -7 expression in vitro and therapeutic regression of experimental endometriosis in vivo by a novel nonsteroidal progesterone receptor agonist, tanaproget. J Clin Endocrinol Metab. 2006;91:155460.

23. Lockwood CJ, Krikun G, Hausknecht VA, Papp C, Schatz F. Matrix metalloproteinase and matrix metalloproteinase inhibitor expression in endometrial stromal cells during progestin-initiated decidualization and menstruation-related progestin withdrawal. Endocrinology. 1998;139:4607-13. 
24. Mönckedieck V, Sannecke C, Husen B, Kumbartski M, Kimmig R, Tötsch M, et al. Progestins inhibit expression of MMPs and of angiogenic factors in human ectopic endometrial lesions in a mouse model. Mol Hum Reprod. 2009;15:633-43.

25. Luddi A, Marrocco C, Governini L, Semplici B, Pavone V, Luisi S, et al. Expression of matrix metalloproteinases and their inhibitors in endometrium: High levels in endometriotic lesions. Int J Mol Sci. 2020;21:2840.

26. Gottschalk C, Malberg K, Arndt M, Schmitt J, Roessner A, Schultze D, et al. Matrix metalloproteinases and TACE play a role in the pathogenesis of endometriosis. Adv Exp Med Biol. 2000;477:483-6.

27. Kotronis K, Zafrakas M, Papasozomenou P, Timologou A, Miliaras D, Tarlatzis BC, et al. Protein expression pattern of tissue inhibitor of metalloproteinase-3 (TIMP3) in endometriosis and normal endometrium. Gynecol Endocrinol. 2019;35:1103-6.

28. Zhang J, Cao YJ, Zhao YG, Sang QX, Duan EK. Expression of matrix metalloproteinase-26 and tissue inhibitor of metalloproteinase-4 in human normal cytotrophoblast cells and a choriocarcinoma cell line, JEG-3. Mol Hum Reprod. 2002;8:659-66.

29. Kai HS, Butler GS, Morrison CJ, King AE, Pelman GR, Overall CM. Utilization of a novel recombinant myoglobin fusion protein expression system to characterize the tissue inhibitor of metalloproteinase (TIMP)-4 and TIMP-2 C-terminal domain and tails by mutagenesis. The importance of acidic residues in binding the MMP-2 hemopexin C-domain. J Biol Chem. 2002;277:48696-707.

30. Liu YE, Wang M, Greene J, Su J, Ullrich S, Li H, et al. Preparation and characterization of recombinant tissue inhibitor of metalloproteinase 4 (TIMP-4). J Biol Chem. 1997;272:20479-83. 
1 31. Stracke JO, Hutton M, Stewart M, Pendás AM, Smith B, López-Otin C, et al.

2 Biochemical characterization of the catalytic domain of human matrix

3 metalloproteinase 19. Evidence for a role as a potent basement membrane degrading

$4 \quad$ enzyme. J Biol Chem. 2000;275:14809-16.

5 32. Pilka R, Domanski H, Hansson S, Eriksson P, Casslén B. Endometrial TIMP-4 mRNA

6 is high at midcycle and in hyperplasia, but down-regulated in malignant tumours.

7 Coordinated expression with MMP-26. Mol Hum Reprod. 2004;10:641-50.

8 33. Iwabe T and Harada T. Inflammation and cytokine in endometriosis. Endometriosis.

9 Tokyo: Springer; 2014. p87-106.

14 Acknowledgement

$15 \quad$ Not applicable.

\section{Funding}

17 This study was funded by the Tsuchiya Foundation (Grant ID:2010(26)-21)).

18 Author information

19 Affiliations

\section{Department of Obstetrics and Gynecology, Hiroshima City Asa Hospital}

Hiroshi Honda, Michinori Yamashita, Yumiko Akimoto, Hirotoshi Tanimoto, Mitsue Teramoto, and Hideki Teramoto

Cell-Matrix Frontier Laboratory, Health Administration Center, Hiroshima University Norihisa Nishimichi, Yasuyuki Yokosaki 
Department of Surgery and Palliative Medicine, Fujita Health University School of Medicine

Michinori Yamashita

\section{Sumire Women's Clinic}

Yumiko Akimoto, Hirotoshi Tanimoto

Department of Obstetrics and Gynecology, Sera Central Hospital

Mitsue Teramoto, and Hideki Teramoto

\section{Contributions}

H.H designed the study, carried out most of the experiments and wrote the paper. N.N. assisted with some experiments. M.Y., Y.A., H.T., M.T. and H. Teramoto provided the patients' samples and clinical information. Y.Y. contributed to the data analysis and interpretation. All of the authors reviewed and revised the manuscript.

\section{Corresponding author}

Correspondence to Hiroshi Honda

\section{Ethics declarations}

\section{Ethics approval and consent to participate}

This study was approved by the Ethical Committee of Hiroshima City Asa Hospital, and all experiments were carried out in accordance with relevant guidelines and regulations. All participants provided their written informed consent.

\section{Consent for publication}

Not applicable

\section{Competing interests}

The authors declare that they have no competing interests. 


\section{$3 \quad$ Figure legends}

4

5 Fig. 1. The top 10 canonical pathways that are significantly distinct in the Dienogest 6 group compared to the Control group. The transverse line indicates the threshold of the 7 distinct pathways in the Dienogest group under the condition of $\mathrm{p}<0.05$. 8

9 Fig. 2. Validation of the microarray data and the pathway analysis by real-time RT-PCR. 10 MMP-1, MMP-3, and MMP-10 were significantly decreased and TIMP-4 was 11 significantly increased in the Dienogest group compared to the Control group. 
Table 1. Top 20 genes upregulated in the Dienogest group

\begin{tabular}{ccc}
\hline Probe ID & Gene Symbol & Fold change \\
\hline A_23_P8801 & CYP3A5 & 5.96 \\
A_33_P3422499 & Not identified & 5.48 \\
A_33_P3327673 & COBL & 4.84 \\
A_19_P00318561 & LOC100506516 & 4.70 \\
A_24_P127691 & DNAH14 & 4.40 \\
A_21_P0008435 & XLOC_011016 & 4.27 \\
A_23_P390958 & PCDHB18 & 4.23 \\
A_33_P3290748 & LOC648044 & 4.10 \\
A_33_P3212269 & MAP2K3 & 4.09 \\
A_24_P7965 & ESRRG & 3.95 \\
A_21_P0010916 & XLC_12001548 & 3.92 \\
A_33_P3409518 & TUBBP5 & 3.77 \\
A_23_P120504 & C20orf46 & 3.76 \\
A_21_P0014652 & LOC100507411 & 3.65 \\
A_19_P00330814 & HOTAIR & 3.51 \\
A_33_P3272614 & PPP1R26 & 3.41 \\
A_33_P3268129 & Not identified & 3.37 \\
A_33_P3333156 & C110rf70 & 3.33 \\
A_21_P0014780 & LOC100652747 & 3.20 \\
A_33_P3424062 & KCNF1 & 3.18 \\
\hline
\end{tabular}


Table 2. Top 20 genes downregulated in the Dienogest group

\begin{tabular}{ccc}
\hline Probe ID & Gene symbol & Fold change \\
\hline A_23_P1452 & NPFFR1 & -6.53 \\
A_23_P313550 & SLC25A41 & -5.84 \\
A_23_P420281 & PRKCB & -5.71 \\
A_23_P329768 & GREB1 & -5.61 \\
A_33_P3376273 & GK & -4.77 \\
A_33_P3409266 & GAFA2 & -4.16 \\
A_32_P32905 & Not identified & -3.67 \\
A_21_P0010584 & XLOC_12_000696 & -3.51 \\
A_33_P3410659 & CLEC12B & -3.49 \\
A_24_P265088 & PDZD4 & -3.45 \\
A_23_P302681 & FIGNL1 & -3.42 \\
A_33_P3262376 & OTUD7A & -3.31 \\
A_24_P395814 & CGB & -3.21 \\
A_33_P3281171 & LOC100130987 & -3.08 \\
A_33_P3270197 & DIS3L2 & -3.06 \\
A_33_P3260342 & NFASC & -2.94 \\
A_21_P001141 & XLOC_000869 & -2.91 \\
A_21_P0010120 & XLOC_013781 & -2.78 \\
A_21_P0001404 & XLOC_000511 & -2.74 \\
A_21_P010200 & XLOC_014056 & -2.72 \\
\hline
\end{tabular}

2

3

4

5

6

7

8

9

10

11

12 
Table 3. Eight pathways to which MMPs are associated among the 20 canonical pathways $\uparrow$ : upregulated in the Dienogest group $\downarrow$ : downregulated in the Dienogest group

\begin{tabular}{|c|c|c|}
\hline Pathway & associated genes & Associated genes \\
\hline Inhibition of Matrix Metalloproteases & 7 & $\begin{array}{l}\text { MMP-1 } \downarrow \text { MMP-3 } \downarrow \text { MMP-10 } \downarrow \text { MMP-12 } \uparrow \\
\text { MMP-25 } \downarrow \text { MMP-27 } \downarrow \text { TIMP-4 } \uparrow\end{array}$ \\
\hline Granulocyte Adhesion and Diapedesis & 13 & 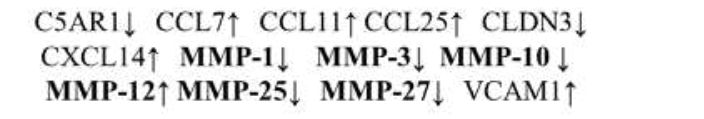 \\
\hline Atherosclerosis Signaling & 11 & 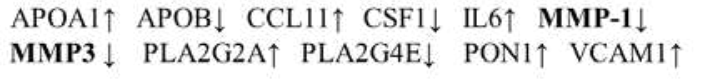 \\
\hline Bladder Cancer Signaling & 7 & $\begin{array}{l}\text { FGF } 13 \uparrow \text { MMP- } 1 \downarrow \text { MMP-3 } \downarrow \text { MMP-10 } \downarrow \\
\text { MMP-12 } \uparrow \text { MPP-25 } \downarrow \text { MMP-27 } \downarrow\end{array}$ \\
\hline HIF $\alpha$ Signaling & 6 & $\begin{array}{l}\text { MMP- } 1 \downarrow \text { MMP-3 } \downarrow \text { MMP-10 } \downarrow \\
\text { MMP-12 } \uparrow \text { MMP-25 } \downarrow \text { MMP-27 } \downarrow\end{array}$ \\
\hline Leukocyte Extravasation Signaling & 9 & $\begin{array}{c}\text { CLDN3 } \downarrow \text { CXCL14 } 1 \text { MMP-1 } \downarrow \text { MMP-3 } \downarrow \text { MMP-10 } \downarrow \\
\text { MMP-12 } \uparrow \text { MMP-25 } \downarrow \text { MMP-27 } \downarrow \text { VCAM } 1 \uparrow\end{array}$ \\
\hline $\begin{array}{l}\text { Role of Macrophages, Fibroblast and Endothelial } \\
\text { Cells in Rheumatoid Arthritis }\end{array}$ & 12 & 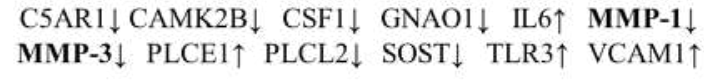 \\
\hline Hepatic Fibrosis /Hepatic Stellate Cell Activation & 8 & 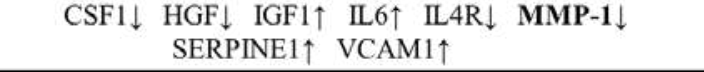 \\
\hline
\end{tabular}


Figures

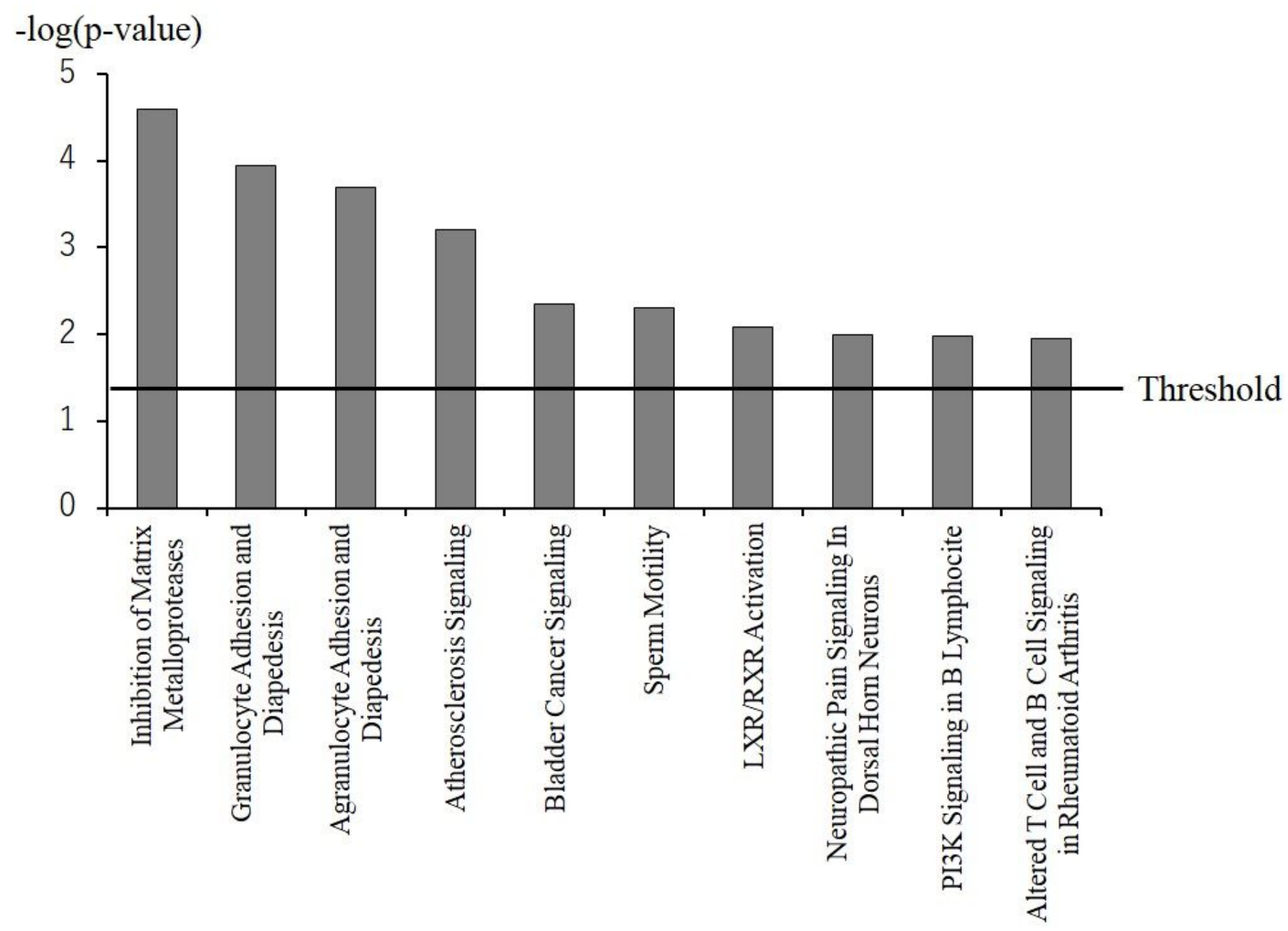

Figure 1

The top 10 canonical pathways that are significantly distinct in the Dienogest group compared to the Control group. The transverse line indicates the threshold of the distinct pathways in the Dienogest group under the condition of $p<0.05$. 


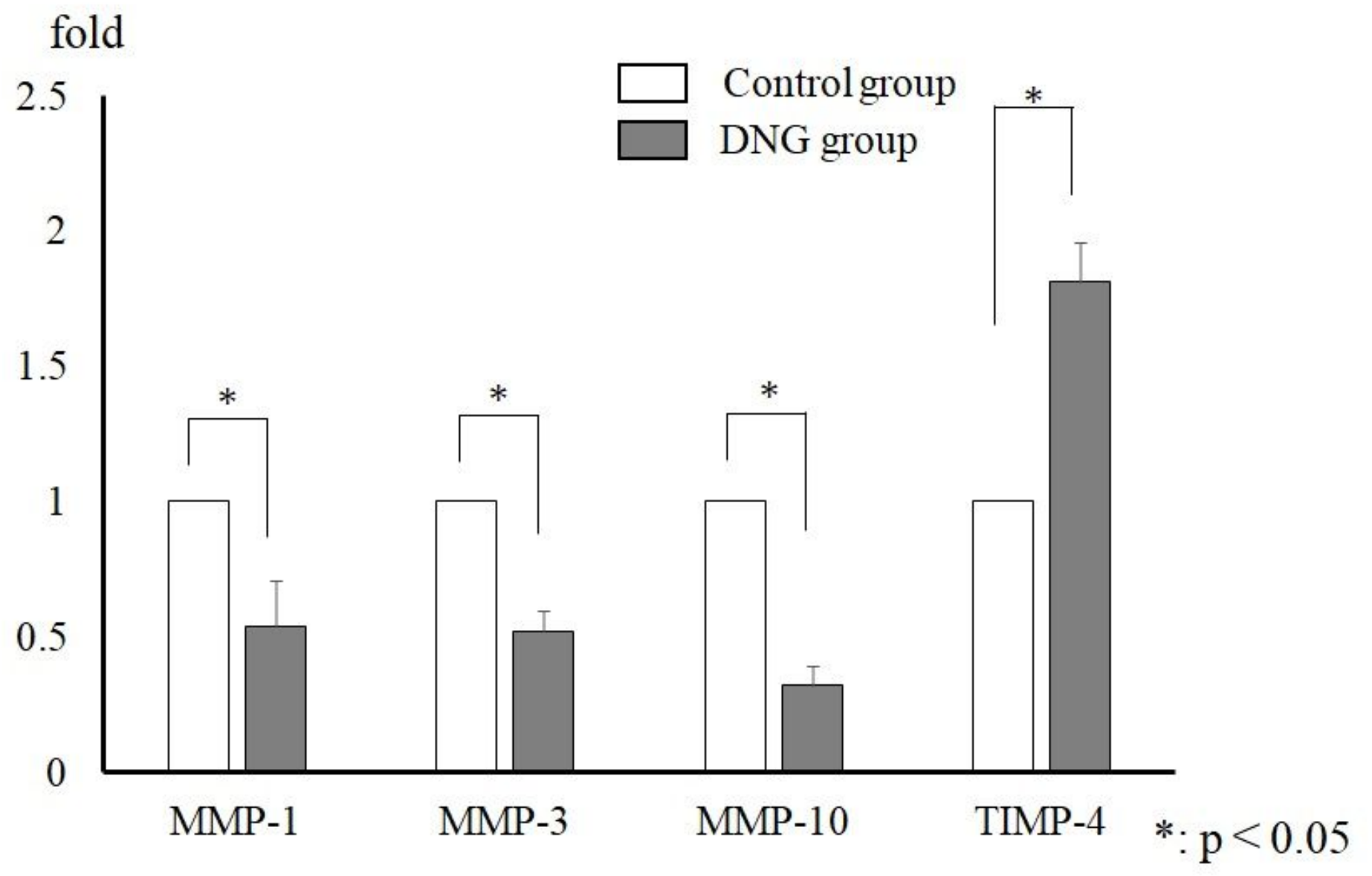

Figure 2

Validation of the microarray data and the pathway analysis by real-time RT-PCR. MMP-1, MMP-3, and MMP-10 were significantly decreased and TIMP-4 was significantly increased in the Dienogest group compared to the Control group. 